\title{
Floquet Fractional Chern Insulators
}

\author{
Adolfo G. Grushin, ${ }^{1,2}$ Álvaro Gómez-León, ${ }^{2}$ and Titus Neupert ${ }^{3}$ \\ ${ }^{1}$ Max-Planck-Institut für Physik komplexer Systeme, 01187 Dresden, Germany \\ ${ }^{2}$ Instituto de Ciencia de Materiales de Madrid, CSIC, Cantoblanco, E-28049 Madrid, Spain \\ ${ }^{3}$ Princeton Center for Theoretical Science, Princeton University, Princeton, New Jersey 08544, USA
}

(Received 25 November 2013; published 18 April 2014)

We show theoretically that periodically driven systems with short range Hubbard interactions offer a feasible platform to experimentally realize fractional Chern insulator states. We exemplify the procedure for both the driven honeycomb and the square lattice, where we derive the effective steady state band structure of the driven system by using the Floquet theory and subsequently study the interacting system with exact numerical diagonalization. The fractional Chern insulator state equivalent to the $1 / 3$ Laughlin state appears at 7/12 total filling (1/6 filling of the upper band). The state also features spontaneous ferromagnetism and is thus an example of the spontaneous breaking of a continuous symmetry along with a topological phase transition. We discuss light-driven graphene and shaken optical lattices as possible experimental systems that can realize such a state.

DOI: 10.1103/PhysRevLett.112.156801

Fractional Chern insulators (FCIs) have been discovered numerically in lattice models of two-dimensional electronic systems [1-5]. They emerge as the ground state of repulsively interacting fermions that partially fill Bloch bands with a nontrivial topological attribute, namely, a nonvanishing Chern number [6]. The time-reversal symmetry (TRS) breaking electronic hopping integrals on the lattice, that are responsible for the band topology, take the role played by the strong magnetic field in the fractional quantum Hall effect. FCIs show that fractional quantum Hall states can appear more generically than previously assumed and do not rely on the specific energetical or analytical properties of Landau levels.

For a system to support a FCI ground state, the energetics have to satisfy specific conditions. For example, if the topological band is spectrally flat $[1,7,8]$ or the energy scale of the repulsive interaction exceeds the energy scales of the band [9], FCIs are favored. This is why the experimental discovery of FCIs is still a formidable experimental challenge, despite the recent experimental realization of its "noninteracting" parent band structure, the Chern insulator or anomalous quantum Hall effect [10]. Needed are systems with a large amount of tunability, to meet both the topological and energetical requirements. Ultracold atomic gases in optical lattices [11], artificial graphene [12], photonic crystals [13], and light-driven solid state systems [14-16] are such tunable platforms, all of which have been shown to potentially host topological band structures. In particular, it has been proven by several theoretical works that circularly polarized light allows us to open a gap at the Dirac cones of the honeycomb lattice, leading to a topologically nontrivial state characterized by chiral edge states $[15,17-19]$. The resulting periodically driven steady state is described by using the Floquet theory and is thus called
PACS numbers: 73.43.-f, 05.30.-d, 05.70.Ln, 71.10.Hf

a Floquet Chern insulator. The key ingredients for its emergence are the critical nature of the Dirac electrons in graphene and the time-reversal symmetry breaking provided by the nonlinearly polarized light [19].

In this Letter, we show that periodically driven systems offer a novel tunable platform to realize robust FCI states experimentally. We shall focus first on the periodically driven honeycomb lattice, a system that can be implemented physically in light-driven graphene or shaken optical lattices [11,20,21] (see Fig. 1). By means of numerical exact diagonalization we show that such a system at 7/12 total filling, when it is periodically driven

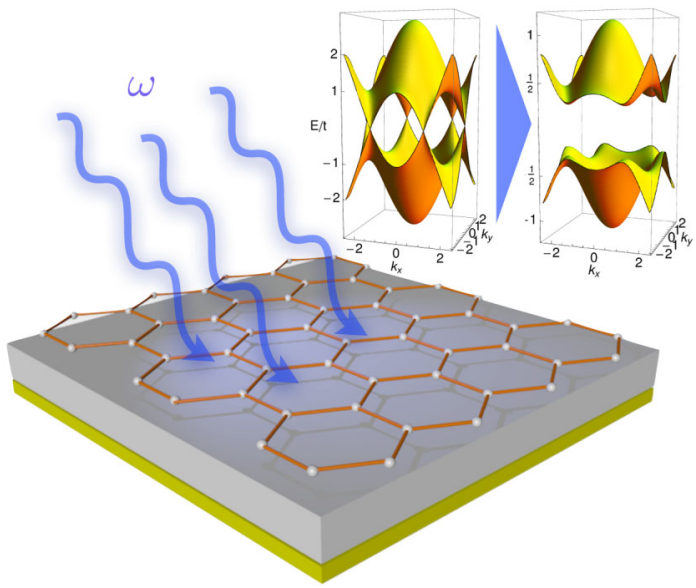

FIG. 1 (color online). A graphene flake is irradiated with light of frequency $\omega$, while a gate voltage is applied via a back gate (yellow) to change the band filling. In the high-frequency regime, the incident light changes the single-particle band structure into an effective Floquet band structure that acquired a gap at the Dirac points (shown are the Floquet bands for the electric field configuration $A_{x}=A_{y}=1.7, \phi=\pi / 2$, and $\left.\omega=10 t_{1}\right)$. 
by circular polarization and high intensity, realizes a ferromagnetic FCI steady state, which we call a Floquet fractional Chern insulator (FFCI). The FFCI state is characterized by a threefold topological ground state degeneracy and a contribution to the Hall conductivity of $\sigma_{H}=\frac{1}{3}\left(e^{2} / h\right)$. Furthermore, we prove that the full $\mathrm{SU}(2)$ spin-rotation symmetry of the model Hamiltonian (in the case of driven graphene, the light field does not couple to the spin and the spin-orbit coupling is negligible) is spontaneously broken for a ferromagnetic steady state, and gapless magnon excitations emerge, coexisting with the FFCI ground state. In addition, we will show that the FFCI state we find is remarkably robust, since it appears for different types of polarizations. The external parameters of the driven field can therefore be used to optimize externally the FFCI state. We conclude by evidencing that the FFCI state appears generically when driving systems that feature critical Dirac fermions, by showing its emergence also in the $\pi$-flux model on the square lattice [22]. We emphasize that we do not rely on a mean-field approximation to obtain these results [23].

Floquet approach for the ac driven honeycomb lattice.We model irradiated monolayer graphene by considering spinful fermions that populate a honeycomb lattice $\Lambda$ and interact repulsively via their on-site $(U)$ and nearest-neighbor $(V)$ electronic densities $n_{i, \sigma}$, for $i \in \Lambda$ and $\sigma=\uparrow, \downarrow$ :

$$
\begin{aligned}
H(\tau) & :=H_{0}(\tau)+H_{\mathrm{int}}, \\
H_{\mathrm{int}} & :=U \sum_{i} n_{i, \uparrow} n_{i, \downarrow}+V \sum_{\langle i, j\rangle} \sum_{\sigma, \sigma^{\prime}} n_{i, \sigma} n_{j, \sigma^{\prime}} .
\end{aligned}
$$

For the single-particle Hamiltonian $H_{0}(\tau)$, we adopt the convention used in Refs. [1,24]. The time dependence is induced via the electromagnetic vector potential $\mathbf{A}(\tau, \phi)=$ $\left(A_{x} \sin (\omega \tau), A_{y} \sin (\omega \tau+\phi), 0\right)^{\top}$ of the external field, where $\omega$ is the frequency of the driving, $\phi$ is the phase difference, $A_{i}=e \mathcal{E}_{i} a / \omega m_{e}, e$ is the electron charge, $m_{e}$ is its mass, $a$ is the lattice spacing, and $\mathcal{E}_{i}$ is the $i$ th component of the driving field. Within perturbation theory to order $1 / \omega$ considered in this work, the ac field affects only the singleparticle Hamiltonian $H_{0}(\tau)$, while the density-density interaction remains as in the undriven case. The reason is that for a density-density interaction, which commutes with the position operator to which the time-dependent electric field couples, further $n$-body terms in the effective Hamiltonian are generated only at the order of $U^{n-1} / \omega^{n}$ in perturbation theory.

Floquet theory provides a powerful formalism to study periodically driven systems. It allows us to easily obtain effective time evolution operators, especially in the regime where the driving frequency is the dominant energy scale [25]. The single-particle Hamiltonian, which enters the time-dependent Schrödinger equation, can be obtained by following Ref. [19], as we explain in the Supplemental Material [26], and has the Fourier decomposition

$$
H_{0, \mathbf{k}}^{q}=\left(\begin{array}{cc}
0 & \left(\rho_{\mathbf{k}}^{-q}\right)^{*} \\
\rho_{\mathbf{k}}^{q} & 0
\end{array}\right), \quad \rho_{\mathbf{k}}^{q}=\sum_{j} t_{j, q}^{\mathrm{F}} e^{i \mathbf{k} \cdot \mathbf{a}_{j}},
$$

where $q \in \mathbb{Z}$ labels the Fourier component in frequency space and the hopping integrals are given by $t_{1, q}^{\mathrm{F}}=t_{1} J_{q}\left(A_{y}\right) e^{i q \phi}, \quad t_{2, q}^{\mathrm{F}}=t_{1} J_{q}\left(A_{+}\right) e^{i q \Psi_{+}}, \quad$ and $\quad t_{3, q}^{\mathrm{F}}=$ $t_{1} J_{-q}\left(A_{-}\right) e^{-i q \Psi_{-}}$. Here, $t_{1}$ is the nearest-neighbor hopping integral for fermions on the honeycomb lattice, and $J_{q}(A)$ denotes the Bessel functions of the first kind. The arguments of the Bessel functions contain the explicit electric field configuration $A_{ \pm}=\sqrt{\left(3 A_{x}^{2} / 4\right)+\left(A_{y}^{2} / 4\right) \pm(\sqrt{3} / 2) A_{x} A_{y} \cos (\phi)}$, and the phase factors are given by $\Psi_{ \pm}=\arctan \left\{A_{y} \sin (\phi) /\right.$ $\left.\left[\sqrt{3} A_{x} \pm A_{y} \cos (\phi)\right]\right\}$. Finally, we define $\mathbf{a}_{1}=(0,0)$, $\mathbf{a}_{2}=(\sqrt{3}, 3) / 2$, and $\mathbf{a}_{3}=(-\sqrt{3}, 3) / 2$ as the unit cell vectors of the honeycomb lattice.

In this work we focus on the high-frequency regime $\left(\omega \gg t_{1}\right)$, where the system dynamics can be accurately described by a static effective Hamiltonian, which can be expanded order by order in $t_{1} / \omega$. To zeroth order in $t_{1} / \omega$, the hopping integrals are renormalized by zeroth-order Bessel functions only, without TRS breaking terms. To first order in $t_{1} / \omega$, corrections to the effective Hamiltonian must be considered due to hybridization with the nearest Floquet bands. Importantly, for nonlinear field polarization, TRS is broken by these corrections, a prerequisite to realize a Floquet Chern insulator. The effective Hamiltonian for long-time dynamics is then defined by including higher Fourier components of the Hamiltonian, leading to the $2 \times 2$ Floquet-Bloch Hamiltonian (for details see the Supplemental Material):

$$
\begin{aligned}
H_{\mathrm{eff}, \mathbf{k}}= & H_{0, \mathbf{k}}^{0}-\frac{1}{\omega}\left(\left[H_{0, \mathbf{k}}^{0}, H_{0, \mathbf{k}}^{-1}\right]-\left[H_{0, \mathbf{k}}^{0}, H_{0, \mathbf{k}}^{1}\right]\right. \\
& \left.+\left[H_{0, \mathbf{k}}^{-1}, H_{0, \mathbf{k}}^{1}\right]\right) .
\end{aligned}
$$

Then, the effective single-particle Hamiltonian expressed by using the second-quantized operators $c_{\mathbf{k}, \sigma}^{\dagger}=$ $\left(c_{\mathbf{k}, \sigma, A}^{\dagger}, c_{\mathbf{k}, \sigma, B}^{\dagger}\right)$, that create an electron with momentum $\mathbf{k}$ and spin $\sigma$ in sublattice $A$ and $B$, respectively, reads

$$
H_{\mathrm{eff}, \mathbf{k}}:=\sum_{\mathbf{k} \in \mathrm{BZ}} \sum_{\sigma=\uparrow, \downarrow} c_{\mathbf{k}, \sigma}^{\dagger} H_{\mathrm{eff}, \mathbf{k}} c_{\mathbf{k}, \sigma} .
$$

The Hamiltonian (4) has two pairs of spin-degenerate bands that touch in two Dirac points for $t_{1} / \omega \rightarrow 0$. The correction to first order in $t_{1} / \omega$ in the $2 \times 2$ Floquet-Bloch Hamiltonian $H_{\text {eff, } \mathbf{k}}$ is proportional to the third Pauli matrix $\sigma_{z}$. Because of the TRS breaking, it can thus potentially open a Haldane-type gap [6] in the spectrum, so that the resulting spin-degenerate bands can acquire a Chern number $c_{1}= \pm 1$ for each spin species. The phase diagram 


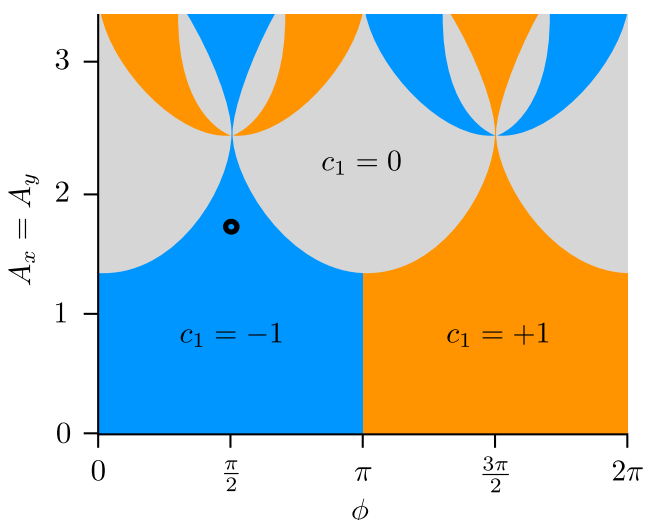

FIG. 2 (color online). Topological phase diagram of periodically driven graphene at a high-frequency regime $\left(\omega=10 t_{1}\right)$ for circularly polarized light. The phase difference $\phi$ and the field amplitude $A_{x}=A_{y}$ allow us to tune the Chern number of the lower spin-degenerate bands of the effective Floquet-Bloch Hamiltonian (3) between $c_{1}=-1$ and $c_{1}=+1$. The black dot indicates the parameter values for which exact diagonalization calculations are presented in Figs. 3 and 4.

for different externally tunable parameters is shown in Fig. 2.

It is worth emphasizing that, in the high-frequency limit relevant for this work, the Hamiltonian (4) is a timeindependent effective Hamiltonian that governs stroboscopic evolution. Therefore, it allows us to fill the bands as in the case of time-independent systems, avoiding dealing with the electronic occupancy of Floquet sidebands.

Exact diagonalization results. - We are now going to show that the ground state of the Floquet Hamiltonian $H_{\text {eff }}+H_{\text {int }}$, and with this the steady state of the driven Hamiltonian (1), can be tuned into a FFCI by controlling (i) the filling of the system with fermions and (ii) the amplitude and phase of the driving field. The emergence of a FFCI depends crucially on the ratios between the energy scales of the single-particle band gap $\Delta$, the single-particle band width $W$, and the repulsive electron-electron interactions, where mathematical band flatness is not always the optimal choice [28]. In graphene, the interaction parameters are given by $U=3 t$ and $V=2 t$ [29]. We neglect furtherrange order interactions, which in principle could lead to additional FCI states at different filling fractions [2]. Choosing incident light with amplitude $A_{x}=A_{y}=1.7$ and the phase shift $\phi=\pi / 2$, for example, results in an $H_{\text {eff }}$ with $\Delta / W=0.6$. We study the system above half filling, so that the upper spin-degenerate band of $H_{\text {eff }}$ is partially filled. Given the size of the single-particle gap $\Delta$, it is reasonable to approximate the states in the lower band to be occupied with probability one, even in the interacting many-body ground state [9]. We thus ignore those singleparticle states and project the degrees of freedom of the system to the upper spin-degenerate band of $H_{\text {eff }}$ by means of the projector $P$ and to study the Hamiltonian

$$
H_{\text {proj }}=P H_{\text {eff }} P+P H_{\text {int }} P \text {. }
$$

Previous studies have shown that FCI ground states equivalent to the $1 / \mathrm{m}$ Laughlin state of the fractional quantum Hall effect in Landau levels emerge quite generically if a flat band with Chern number $c_{1}=1$ is populated with spinless fermions at a filling $\nu=1 / m, m \in \mathbb{Z}$ $[1-3,24]$. In contrast, we are considering a dispersionful band with Chern number $c_{1}=1$ that is partially filled with spinful fermions in such a way that the Hamiltonian is $\mathrm{SU}(2)$ spin-rotation symmetric. In anticipation of a spontaneous breaking of the SU(2) symmetry by the many-body ground state, we therefore study the system at $\nu=1 /(2 m)$ filling to obtain a FFCI that is equivalent to a $\nu=1 / m$ Laughlin state. We have performed numerical exact diagonalization of the Hamiltonian (5) on lattices with $L_{x} \times$ $L_{y}=4 \times 3$ and $L_{x} \times L_{y}=4 \times 6$ unit cells with $N=4$ and $N=8$ fermions, respectively, with periodic boundary conditions in place. Good quantum numbers of the many-body states are the total spin $S=0, \ldots, N / 2$, the total spin- $z$ component $S_{z}=-N / 2, \ldots, N / 2$, and the center of mass momentum $Q \in\left[0, L_{x} \times L_{y}-1\right]$. All results and conclusions presented below extend to both lattice sizes, and we focus on the $L_{x} \times L_{y}=4 \times 6$ lattice here, delegating the consistency check with $L_{x} \times L_{y}=4 \times 3$ to the Supplemental Material.

We observe that the low-energy states have both exact and approximate degeneracies: In each of the $(N+1)$ sectors of $S_{z}$, three low-lying states are found which are not exactly, but approximately, degenerate. Each of these three states has an exactly degenerate partner in every other $S_{z}$ sector (see Fig. 3). The total ground state degeneracy that

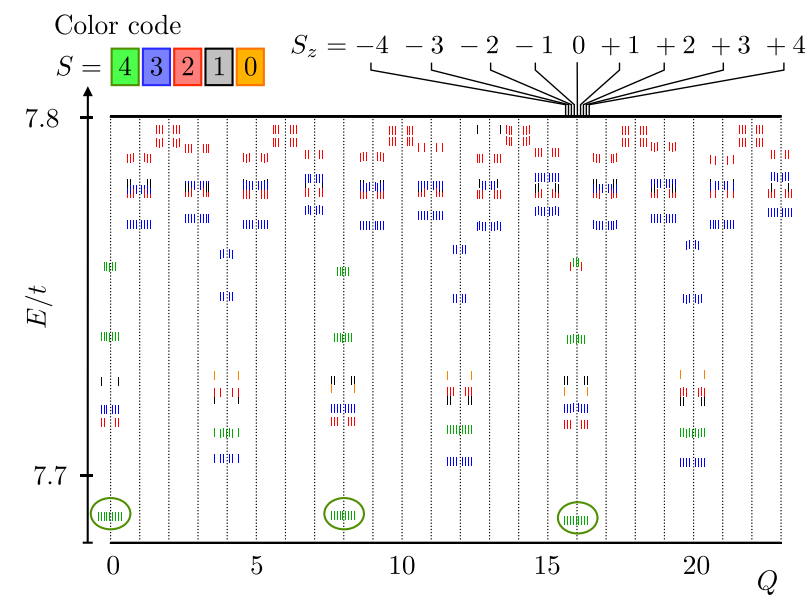

FIG. 3 (color online). Low-lying portion of the energy spectrum of Hamiltonian (5) on a $L_{x} \times L_{y}=4 \times 6$ with $N=8$ particles. Encircled is the ground state manifold with threefold topological (quasi)degeneracy of the fractional Chern insulator and $(N+1=9)$-fold degeneracy as a precursor of spontaneous symmetry breaking toward a ferromagnetic phase in the thermodynamic limit. The good quantum numbers total momentum $Q=k_{x}+L_{x} k_{y}$, total spin $S$, and total spin in $z$ direction $S_{z}$ are indicated. 
we anticipate in the limit of large particle number $N$ is thus $3(N+1)$. As all ground states have the maximum spin $S=N / 2$, we interpret the exact $(N+1)$-fold degeneracy as a finite-size precursor of a spontaneous breaking of the $\mathrm{SU}(2)$ symmetry towards a ferromagnetic ground state in the thermodynamic limit. Both the tower-of-states structure [30] in the spectrum as a function of $S$ [Fig. 4(b)] and the exactness of the degeneracy support this conclusion (the order parameter $S_{z}$ of ferromagnetism commutes with the Hamiltonian, thus rendering the ground state degeneracy exact already for finite systems). In contrast, the threefold approximate degeneracy in each $S_{z}$ sector is of topological origin. It is the $m$-fold topological ground state degeneracy of a $1 / \mathrm{m}$ Laughlin state on the torus in the case $m=3$. The nonlocal Wilson-loop order parameter does not commute with the Hamiltonian, rendering the degeneracy approximate in the finite system. Further supporting arguments that the three low-lying states in each $S_{z}$ sector are indeed topologically ordered FFCI states are the following. (i) By inserting a flux in the torus (which is equivalent to changing the boundary conditions from periodic to twisted [31]), the three states permute and return to their original order after three flux quanta [see Fig. 4(a)]. This evidences charge fractionalization with quasiparticles of charge $e / 3$ and indicates that the topological ground states survive in the thermodynamic limit. (ii) The three states occur at the momentum sectors $Q$ that are predicted by the counting rule of Ref. [3] that is based on a 1/3 Laughlin state. (iii) Any superposition of the three ground states has a nearly constant charge density in position space, which excludes that these states would form a charge-density wave in the thermodynamic limit (see Supplemental Material).
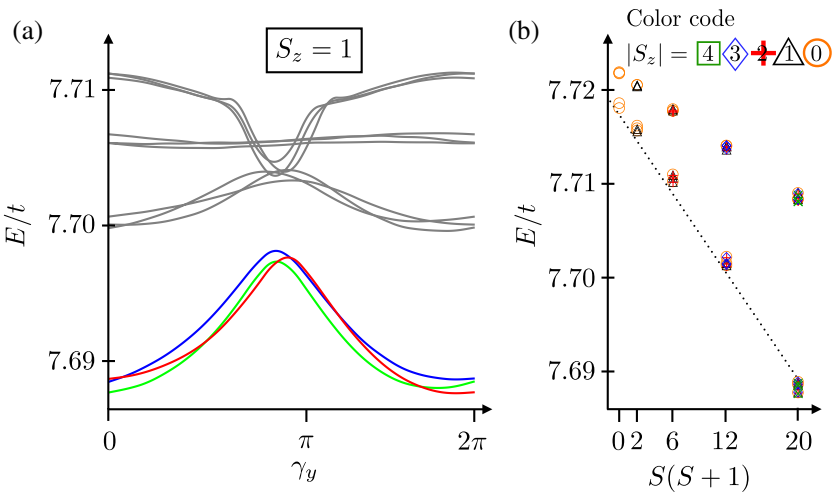

FIG. 4 (color online). (a) Spectral evolution of the energy spectrum of Fig. (3) in the sector with $S_{z}=1$ upon inserting a flux $\gamma_{y}$ into the system, which is synonymous to twisting the boundary conditions in the $y$ direction with a complex phase $e^{\mathrm{i} \gamma_{y}}$. The three fractional Chern insulator ground states evolve independently of the rest of the spectrum and trade places, which signals their topological degeneracy and the charge fractionalization. (b) The energy spectrum of Fig. 3 plotted against the total spin $S(S+1)$ reveals the tower of states which evidence the ferromagnetic nature of the ground state.
From the presented results, we conclude that the steady state of the driven honeycomb lattice with spinful fermions at $7 / 12$ total filling (or $1 / 6$ filling of the band above the Dirac cone) is a ferromagnetic FFCI. This distinct driven topological state has gapped charged excitations but supports gapless spin excitations, namely, ferromagnetic magnons. Remarkably, the state features both conventional order and gapless topological order. Experimentally, its signature is a fractional contribution of $\sigma_{H}=\frac{1}{3}\left(e^{2} / h\right)$ (in addition to the integer contribution of the lower band) defined for the driven system [32].

To certify the robustness of the FFCI state we have also tested its stability under changing some of the conditions discussed above. First, we have investigated its fate upon changing to different light polarizations (using $A_{x} / A_{y}=\sqrt{3}, 1 / \sqrt{3}$ as two examples). The FFCI is still the ground state of the system as long as the Chern number of the noninteracting band is $c_{1}= \pm 1$. Second, we have investigated its appearance on a different lattice system. In particular, we find that for spinless fermions on the $\pi$-flux square lattice [22] with nearest-neighbor repulsive interaction the ground state is also the FFCI whenever $\left(A_{x}=A_{y}, \phi\right)$ are such that the noninteracting bands have $c_{1}= \pm 1$ (see Supplemental Material). Both of these results evidence the ubiquitousness and robustness of the FFCI state.

Possible experimental realizations. - The practical realization of this novel state in graphene possesses two experimental challenges. The first is to reach incident field amplitudes $\mathcal{E}_{x}=\mathcal{E}_{y} \sim \omega \gg t_{1}$. Although it is in principle possible to reach such a regime, today experiments have explored only amplitudes of one order of magnitude lower [33]. However, given the robustness and tunability of the effect, it is conceivable that lower frequencies and field amplitudes can in fact be sufficient to access the FFCI state in the thermodynamic limit. The second experimental issue is reaching the necessary filling factor of graphene's band structure. In particular, the electron density at $1 / 6$ filling of the upper band is of the order of $1 \times 10^{14} \mathrm{e} / \mathrm{cm}^{2}$, still below the van Hove singularity in graphene. Even higher values up to the van Hove singularity have already been reached by chemical doping [34], and there are promising other routes by using, for example, polymer electrolytes [35]. Alternatively, shaken optical lattices $[11,20,21,36]$ can provide a platform to realize the spinless version of the effect with experimentally available amplitudes of the driving field. In this context, time of flight measurements in the spirit of Ref. [37] could be used to distinguish the FFCI phase. Finally, other 2D materials with Dirac electrons such as silicene [38] can potentially be used to tune the band structure parameters and host similar phases.

In summary, we have found that spinful fermions on the honeycomb and $\pi$-flux square lattice, when coupled to external polarized fields, undergo an interaction-driven 
topological phase transition to a FFCI state that features topological order and spontaneous ferromagnetic order. Moreover, the robustness and tunability of the FFCI state, appearing for different parameters, polarizations, and lattices, evidences that periodically driven systems offer an experimentally appealing platform to realize FCI states. Our work thus opens up a promising route to achieve tunable realizations of elusive interacting fermionic and bosonic phases by periodically driving interacting systems.

We acknowledge L. D'Alessio, E. Berg, D. Podolsky, G. Platero, and M. A. H. Vozmediano for useful discussions. Financial support from PIB2010BZ-00512 (A. G. G.), JAE program, MAT 2011-24331, and ITN, Grant No. 234970 (EU) (A. G-L.), and Swiss National Science Foundation (T. N.) is greatly acknowledged.

[1] T. Neupert, L. Santos, C. Chamon, and C. Mudry, Phys. Rev. Lett. 106, 236804 (2011).

[2] D. Sheng, Z.-C. Gu, K. Sun, and L. Sheng, Nat. Commun. 2, 389 (2011).

[3] N. Regnault and B. A. Bernevig, Phys. Rev. X 1, 021014 (2011).

[4] E. J. Bergholtz and Z. Liu, Int. J. Mod. Phys. B 27, 1330017 (2013).

[5] S. A. Parameswaran, R. Roy, and S. L. Sondhi, arXiv:1302.6606.

[6] F. D. M. Haldane, Phys. Rev. Lett. 61, 2015 (1988).

[7] E. Tang, J.-W. Mei, and X.-G. Wen, Phys. Rev. Lett. 106, 236802 (2011).

[8] K. Sun, Z.-C. Gu, H. Katsura, and S. Das Sarma, Phys. Rev. Lett. 106, 236803 (2011).

[9] S. Kourtis, J. W. F. Venderbos, and M. Daghofer, Phys. Rev. B 86, 235118 (2012).

[10] C.-Z. Chang et al., Science 340, 167 (2013).

[11] T. Uehlinger, G. Jotzu, M. Messer, D. Greif, W. Hofstetter, U. Bissbort, and T. Esslinger, Phys. Rev. Lett. 111, 185307 (2013).

[12] K. K. Gomes, W. Mar, W. Ko, F. Guinea, and H. C. Manoharan, Nature (London) 483, 306 (2012).

[13] M. C. Rechtsman, J. M. Zeuner, Y. Plotnik, Y. Lumer, D. Podolsky, F. Dreisow, S. Nolte, M. Segev, and A. Szameit, Nature (London) 496, 196 (2013).

[14] N. H. Lindner, G. Refael, and V. Galitski, Nat. Phys. 7, 490 (2011)

[15] T. Kitagawa, T. Oka, A. Brataas, L. Fu, and E. Demler, Phys. Rev. B 84, 235108 (2011).

[16] P. Delplace, A. Gomez-Leon, and G. Platero, Phys. Rev. B 88, 245422 (2013).

[17] Z. Gu, H. A. Fertig, D. P. Arovas, and A. Auerbach, Phys. Rev. Lett. 107, 216601 (2011).
[18] T. Oka and H. Aoki, Phys. Rev. B 79, 081406 (2009).

[19] A. Gomez-Leon, P. Delplace, and G. Platero, arXiv:1309.5402.

[20] J. Struck, C. Ölschläger, M. Weinberg, P. Hauke, J. Simonet, A. Eckardt, M. Lewenstein, K. Sengstock, and P. Windpassinger, Phys. Rev. Lett. 108, 225304 (2012).

[21] P. Hauke et al., Phys. Rev. Lett. 109, 145301 (2012).

[22] X. G. Wen, F. Wilczek, and A. Zee, Phys. Rev. B 39, 11413 (1989).

[23] In previous work [J. W. F. Venderbos, S. Kourtis, J. van den Brink, and M. Daghofer, Phys. Rev. Lett. 108, 126405 (2012)], a mean-field treatment was employed to obtain a magnetically ordered background, and the resulting band structure was studied at partial filling, where the mean-field approximation is not justified anymore.

[24] Y.-L. Wu, B. A. Bernevig, and N. Regnault, Phys. Rev. B 85, 075116 (2012).

[25] A. Gomez-Leon and G. Platero, Phys. Rev. Lett. 110, 200403 (2013).

[26] See Supplemental Material at http://link.aps.org/ supplemental/10.1103/PhysRevLett.112.156801 for a detailed derivation of the Floquet-Bloch Hamiltonian and further numerical evidence for the FFCI state, which includes Ref. [27].

[27] T. Neupert, L. Santos, C. Chamon, and C. Mudry, Phys. Rev. B 86, 165133 (2012).

[28] A. G. Grushin, T. Neupert, C. Chamon, and C. Mudry, Phys. Rev. B 86, 205125 (2012).

[29] T. O. Wehling, E. Şaşıŏ̆lu, C. Friedrich, A. I. Lichtenstein, M. I. Katsnelson, and S. Blügel, Phys. Rev. Lett. 106, 236805 (2011).

[30] C. Lacroix, P. Mendels, and F. Mila, Introduction to Frustrated Magnetism: Materials, Experiments, Theory (Springer, New York, 2010), Vol. 164.

[31] Q. Niu, D. J. Thouless, and Y.-S. Wu, Phys. Rev. B 31, 3372 (1985).

[32] A. Kundu and B. Seradjeh, Phys. Rev. Lett. 111, 136402 (2013).

[33] S. Tani, F. Blanchard, and K. Tanaka, Phys. Rev. Lett. 109, 166603 (2012).

[34] J. L. McChesney, A. Bostwick, T. Ohta, T. Seyller, K. Horn, J. González, and E. Rotenberg, Phys. Rev. Lett. 104, 136803 (2010)

[35] A. Pachoud, M. Jaiswal, P. K. Ang, K. P. Loh, and B. Oezyilmaz, Europhys. Lett. 92, 27001 (2010).

[36] A. Zenesini, H. Lignier, D. Ciampini, O. Morsch, and E. Arimondo, Phys. Rev. Lett. 102, 100403 (2009).

[37] E. Alba, X. Fernandez-Gonzalvo, J. Mur-Petit, J. K. Pachos, and J. J. Garcia-Ripoll, Phys. Rev. Lett. 107, 235301 (2011).

[38] N. D. Drummond, V. Zólyomi, and V. I. Fal'ko, Phys. Rev. B 85, 075423 (2012). 